\title{
Reverse and Forward Engineering of Local Voltage Control in Distribution Networks
}

\author{
Xinyang Zhou, Masoud Farivar, Zhiyuan Liu, Lijun Chen and Steven Low
}

\begin{abstract}
The increasing penetration of renewable and distributed energy resources in distribution networks calls for realtime and distributed voltage control. In this paper we investigate local Volt/VAR control with a general class of control functions, and show that the power system dynamics with non-incremental local voltage control can be seen as a distributed algorithm for solving a well-defined optimization problem (reverse engineering). The reverse engineering further reveals a fundamental limitation of the non-incremental voltage control: the convergence condition is restrictive and prevents better voltage regulation at equilibrium. This motivates us to design two incremental local voltage control schemes based on the subgradient and pseudo-gradient algorithms respectively for solving the same optimization problem (forward engineering). The new control schemes decouple the dynamical property from the equilibrium property, and have much less restrictive convergence conditions. This work presents another step towards developing a new foundation-network dynamics as optimization algorithms-for distributed real-time control and optimization of future power networks.
\end{abstract}

Index Terms-Distributed control and optimization, voltage regulation, network dynamics as optimization algorithms, reverse and forward engineering, power networks.

\section{INTRODUCTION}

Traditionally, given the predictable and relatively slow changes in power demand, capacitor banks and load tap changers are switched a few times per day to regulate the voltages in distribution systems; see, e.g., [1], [2]. However, with the increasing penetration of renewable energy resources such as photovoltaic (PV) and wind turbines in both residential and commercial settings [3], [4], the intermittent and fastchanging renewable energy supply introduces rapid fluctuations in voltages that are beyond the capability of those traditional voltage regulation schemes and thus calls for new voltage control paradigms.

X. Zhou is with the Power System Engineering Department, National Renewable Energy Laboratory, Golden, CO 80401, USA (Email: xinyang.zhou@nrel.gov).

Z. Liu and L. Chen are with the Department of Computer Science, University of Colorado, Boulder, CO 80309, USA (Emails: \{zhiyuan.liu, lijun.chen\}@colorado.edu).

M. Farivar is with Google (Email: mfarivar@gmail.com).

S. Low is with the Division of Engineering and Applied Science, California Institute of Technology, Pasadena, CA 91125, USA (Email: slow@caltech.edu).

This work was partly supported by NREL under subcontracts APUP UGA-0-41026-120 and APUP UGA-0-41026-107, NSF through grants CCF 1637598, ECCS 1619352 and CPS ECCS 1739355, and DOE under contract No. DE-EE-0007998.

Preliminary results of this paper have been presented at IEEE Conference on Decision and Control, Florence, Italy, 2013 [53], IEEE International Conference on Smart Grid Communications, Miami, FL, 2015 [54], and Annual Allerton Conference on Communication, Control, and Computing, Allerton, IL, 2015 [55].

\section{A. Inverter-Based Voltage Regulation}

Even though the current IEEE Standard 1547 [5] requires distributed generation to operate at unity power factor, inverters can readily adjust real and reactive power outputs to stabilize voltages and cope with fast time-varying conditions. Indeed, the IEEE Standards group is actively exploring a new inverter-based Volt/VAR control. Unlike the capacity banks or tap changers, inverters can push and pull reactive power much faster, in a much finer granularity and with low operation cost, enabling real-time distributed control that is needed for the future power grid with a large number of renewable and distributed energy resources.

Inverter-based voltage regulation has been studied extensively in the literature. Related work roughly falls into the following categories:

1) Centralized control: By collecting all the required information and computing a global optimal power flow (OPF) problem, a central controller determines optimal set-points for the whole system; see, e.g., [6]-[10]. Centralized control can incorporate general objectives and operational constraints, but suffers from considerable communication overhead and long computation time especially when the size of the system is large. Therefore, it usually cannot provide fast control.

2) Distributed control: For OPF problems with certain structures, one can design algorithms to distribute the computation with coordinating communication, which is conducted either between a central controller and agents in a hierarchical way, e.g., [11]-[18], or among neighborhoods of individual agents without any central controller, e.g., [19]-[24]. Given the required communication infrastructure, the scheme based on distributed OPF algorithm can provide scalable voltage control.

3) Local control: Based only on local information, local voltage control provides fast response and, without the need of communication, allows simple and scalable implementation; see, e.g., [25]-[32].

In this paper, we focus on the analysis and design of local voltage control. Characterization of local control, especially system-wide properties arising from the interaction between local controls, is challenging. In the literature, the work such as [31], [33] lacks analytical characterization. Other work such as [29], [30], [34], [35] provides stability analysis but lacks systemwide performance characterization of the equilibrium point. There is work that provided rigorous performance analysis for stability and system-wide performance of local voltage control, but are subject to control functions of a particular type, e.g., linear control functions without deadband [26], [36] and quadratic control functions [25]. 
This paper is the extended version of our preliminary work [53]-[55]. In [53] we study a general class of local voltage control algorithms and characterize their global performance as well as convergence properties, and in [54], [55] we propose two different local voltage control schemes based on the subgradient and pseudo-gradient algorithms with better convergence properties. This paper significantly improves upon our preliminary work. In particular, 1) we have adopted new mathematical techniques to re-do all convergence proofs to explicitly handle the non-differentiability issue caused by the deadband in control, 2) we have enriched the characterization of the equilibrium based on a new characterization of the inverse reactance matrix, 3) we have added new numerical results to complement the analytical results, and importantly 4) we have laid out a general reverse and forward engineering framework for systematic analysis and design of local voltage control; see the next subsection for more detail. Related work also includes [32] that shows the uniqueness of equilibrium of local voltage control as in [53] but using a different technique and does not provide convergence proof, and [34], [35] that consider the pseudo-gradient algorithm as in [55] but without deadband in control and do not have a characterization of the equilibrium as the optimum of an optimization problem.

\section{B. Reverse and Forward Engineering}

Different from other work, in this paper, we consider local voltage control with general monotone control functions, and seek a principled way to guide systematic analysis and design of local voltage control with a global perspective through the approach of reverse and forward engineering. We first develop models to understand the systemwide properties arising from the interaction between local controls, in particular, whether the power system dynamics with existing controls can be interpreted as distributed algorithms for solving certain optimization problems, i.e., network dynamics as optimization algorithms. We then leverage the insights obtained from the reverse engineering to design new local voltage control schemes according to distributed algorithms for solving the resulting optimization problem (or its variant that incorporates new design objective and/or constraints).

Specifically, we first lay out a general framework for reverse engineering power system dynamics with non-incremental local voltage control with general control functions, and show that it can be seen as a distributed algorithm for solving a well-defined optimization problem. We characterize the condition under which the dynamical system converges, which is however very restrictive and prevents better voltage regulation at the equilibrium (or optimum): aggressive control functions are preferred for better voltage regulation at equilibrium, while less aggressive ones are preferred for convergence. We are therefore motivated to find a way to decouple the dynamical property from the equilibrium property.

Notice that the optimization-based model does not only provide a way to characterize the equilibrium and establish the convergence of power system dynamics with local control (i.e., reverse engineering) but also suggests a principled way to engineer the control to achieve the desired property (i.e., forward engineering). In particular, new control schemes with better dynamical properties can be designed based on different optimization algorithms for solving the same optimization problem. Accordingly, we propose an incremental local voltage control scheme based on the (sub)gradient algorithm for solving the same optimization problem. This new control scheme decouples the equilibrium property and the convergence property and has much less restrictive convergence condition. However, it converges to only within a small neighborhood of the equilibrium. Furthermore, it requires computing the inverse of the control function, which may incur considerable computation overhead. We thus propose another incremental local voltage control scheme based on a pseudogradient algorithm that has better convergence property and simpler implementation than the (sub)gradient control while achieving the same equilibrium.

Similar ideas of reverse and forward engineering based on the perspective of network dynamics as optimization algorithms have been applied to distributed real-time frequency control of power systems, e.g., [37]-[43], as well as synchronization of networks of coupled oscillators [44]. This paper presents another step towards developing a new foundation network dynamics as optimization algorithms - for distributed real-time control and optimization of future power networks.

The rest of the paper is organized as follows. Section II describes the system model and introduces the non-incremental local voltage control. Section III investigates the equilibrium and dynamical properties of the non-incremental local control by reverse engineering. Section IV proposes two incremental local voltage control schemes that decouple the equilibrium and convergence properties and have much less restrictive convergence conditions. Section V provides numerical examples to complement the theoretical analysis, and Section VI concludes the paper.

\section{Network Model and Local Voltage Control}

Consider a tree graph $\mathcal{G}=\{\mathcal{N} \cup\{0\}, \mathcal{L}\}$ that represents a radial distribution network consisting of $n+1$ buses and a set $\mathcal{L}$ of directed lines between these buses whose directions are pointing away from bus 0 . Bus 0 is the substation bus (slack bus) and is assumed to have a fixed voltage of $v_{0}=1$ p.u. Let $\mathcal{N}:=\{1, \ldots, n\}$. Due to the tree topology, we have the cardinality of the line set $|\mathcal{L}|=n$. For each bus $i \in \mathcal{N}$, denote by $\mathcal{L}_{i} \subseteq \mathcal{L}$ the set of lines on the unique path from bus 0 to bus $i, p_{i}^{c}$ and $p_{i}^{g}$ the real power consumption and generation respectively, and $q_{i}^{c}$ and $q_{i}^{g}$ the reactive power consumption and generation respectively. Let $v_{i}$ be the magnitude of the complex voltage (phasor) at bus $i$. For each line $(i, j) \in \mathcal{L}$, denote by $r_{i j}$ and $x_{i j}$ its resistance and reactance, and $P_{i j}$ and $Q_{i j}$ the sending-end real and reactive power from bus $i$ to bus $j$. Let $\ell_{i j}$ denote the squared magnitude of the complex branch current (phasor) from bus $i$ to bus $j$. We summarize some of the notations used in this paper in Section II-A. 


\section{A. Notation}

\begin{tabular}{|c|c|}
\hline $\mathcal{N}$ & set of buses excluding bus $0, \mathcal{N}:=\{1, \ldots, n\}$ \\
\hline $\mathcal{L}$ & set of power lines \\
\hline $\mathcal{L}_{i}$ & set of lines from bus 0 to bus $\mathrm{i}$ \\
\hline$p_{i}^{c}, q_{i}^{c}$ & real, reactive power consumption at bus $i$ \\
\hline $\begin{array}{l}q_{i}^{g}, q_{i}^{g} \\
P_{i j}, Q_{i j}\end{array}$ & $\begin{array}{l}\text { real, reactive power generation at bus } i \\
\text { real and reactive power flow from } i \text { to } j\end{array}$ \\
\hline$r_{i j}, x_{i j}$ & resistance and reactance of line $(i, j)$ \\
\hline & magnitude of complex voltage at bus $i$ \\
\hline$\ell_{i j}$ & $\begin{array}{l}\text { squared magnitude of complex current of } \\
\text { line }(i, j)\end{array}$ \\
\hline & $\begin{array}{l}\text { feasible power set of inverter } i \text {; } \Omega:=\chi_{i=1}^{n} \Omega_{i} \\
\text { projection onto set } \Omega_{i}\end{array}$ \\
\hline$\sigma_{\max }()$ & maximum singular value of a matrix \\
\hline$\lambda_{\max }()$ & maximum eigenvalue of a matrix \\
\hline $\bar{\alpha}_{i}$ & $\begin{array}{l}\text { upper-bound of the (sub)derivative } d f_{i}\left(v_{i}\right) / d v_{i} \\
\bar{A}:=\operatorname{diag}\left\{\bar{\alpha}_{1}, \ldots, \bar{\alpha}_{n}\right\} \in \mathbb{S}_{++}^{N}\end{array}$ \\
\hline
\end{tabular}

A quantity without subscript is usually a vector with appropriate components defined earlier, e.g., $v:=\left(v_{i}, i \in \mathcal{N}\right), q^{g}:=$ $\left(q_{i}^{g}, i \in \mathcal{N}\right)$.

\section{B. Linearized Branch Flow Model}

We adopt the following branch flow model introduced in [1], [2] (DistFlow equations) to model a radial distribution system:

$$
\begin{aligned}
P_{i j} & =p_{j}^{c}-p_{j}^{g}+\sum_{k:(j, k) \in \mathcal{L}} P_{j k}+r_{i j} \ell_{i j}, \\
Q_{i j} & =q_{j}^{c}-q_{j}^{g}+\sum_{k:(j, k) \in \mathcal{L}} Q_{j k}+x_{i j} \ell_{i j}, \\
v_{j}^{2} & =v_{i}^{2}-2\left(r_{i j} P_{i j}+x_{i j} Q_{i j}\right)+\left(r_{i j}^{2}+x_{i j}^{2}\right) \ell_{i j}, \\
\ell_{i j} v_{i}^{2} & =P_{i j}^{2}+Q_{i j}^{2} .
\end{aligned}
$$

Following [45] we assume that the terms involving $\ell_{i j}$ are zero for all $(i, j) \in \mathcal{L}$ in (1). This approximation neglects the higher-order real and reactive power loss terms. Since losses are typically much smaller than power flows $P_{i j}$ and $Q_{i j}$, it only introduces a small relative error, typically on the order of $1 \%$. We further assume that $v_{i} \approx 1, \forall i$ so that we can set $v_{j}^{2}-v_{i}^{2}=2\left(v_{j}-v_{i}\right)$ in equation (1c). This approximation introduces a small relative error of at most $0.25 \%$ if there is a $5 \%$ deviation in voltage magnitude.

With the above approximations (1) is simplified to the following linear model:

$$
\begin{aligned}
P_{i j} & =\sum_{k \in \beta(j)}\left(p_{k}^{c}-p_{k}^{g}\right), \\
Q_{i j} & =\sum_{k \in \beta(j)}\left(q_{k}^{c}-q_{k}^{g}\right), \\
v_{i}-v_{j} & =r_{i j} P_{i j}+x_{i j} Q_{i j},
\end{aligned}
$$

where $\beta(j)$ is the set of all descendants of bus $j$ including bus $j$ itself, i.e., $\beta(j)=\left\{i \mid \mathcal{L}_{j} \subseteq \mathcal{L}_{i}\right\}$. This yields an explicit solution for $v_{i}$ in terms of $v_{0}$ (which is given and fixed):

$$
\begin{aligned}
& v_{0}-v_{i}=\sum_{(j, k) \in \mathcal{L}_{i}} r_{j k} P_{j k}+\sum_{(j, k) \in \mathcal{L}_{i}} x_{j k} Q_{j k} \\
= & \sum_{(j, k) \in \mathcal{L}_{i}} r_{j k}\left(\sum_{h \in \beta(k)}\left(p_{h}^{c}-p_{h}^{g}\right)\right)+\sum_{(j, k) \in \mathcal{L}_{i}} x_{j k}\left(\sum_{h \in \beta(k)}\left(q_{h}^{c}-q_{h}^{g}\right)\right) \\
= & \sum_{j \in \mathcal{N}}\left(p_{j}^{c}-p_{j}^{g}\right)\left(\sum_{(h, k) \in \mathcal{L}_{i} \cap \mathcal{L}_{j}} r_{h k}\right)+\sum_{j \in \mathcal{N}}\left(q_{j}^{c}-q_{j}^{g}\right)\left(\sum_{(h, k) \in \mathcal{L}_{i} \cap \mathcal{L}_{j}} x_{h k}\right) \\
= & \sum_{j \in \mathcal{N}} R_{i j}\left(p_{j}^{c}-p_{j}^{g}\right)+\sum_{j \in \mathcal{N}} X_{i j}\left(q_{j}^{c}-q_{j}^{g}\right),
\end{aligned}
$$

where

$$
R_{i j}:=\sum_{(h, k) \in \mathcal{L}_{i} \cap \mathcal{L}_{j}} r_{h k}, \quad X_{i j}:=\sum_{(h, k) \in \mathcal{L}_{i} \cap \mathcal{L}_{j}} x_{h k} .
$$

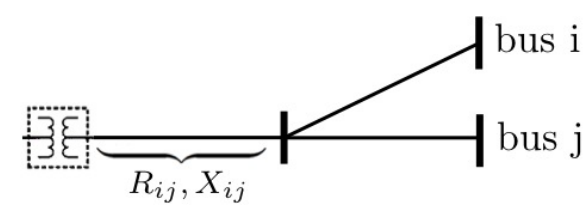

Fig. 1. $\mathcal{L}_{i} \cap \mathcal{L}_{j}$ for two arbitrary buses $i, j$ in the network and the corresponding mutual voltage-to-power-injection sensitivity factors $R_{i j}, X_{i j}$.

Fig. 1 gives an illustration of $\mathcal{L}_{i} \cap \mathcal{L}_{j}$ for two arbitrary buses $i$ and $j$ in a radial network and the corresponding $R_{i j}$ and $X_{i j}$. Since

$$
\begin{aligned}
& R_{i j}=\frac{\partial v_{i}}{\partial p_{j}^{g}}=-\frac{\partial v_{i}}{\partial p_{j}^{c}}, \\
& X_{i j}=\frac{\partial v_{i}}{\partial q_{j}^{g}}=-\frac{\partial v_{i}}{\partial q_{j}^{c}},
\end{aligned}
$$

$R_{i j}, X_{i j}$ are also referred to as the mutual voltage-to-powerinjection sensitivity factors.

Define a resistance matrix $R=\left[R_{i j}\right]_{n \times n}$ and a reactance matrix $X=\left[X_{i j}\right]_{n \times n}$. Both matrices are symmetric. With the matrices $R$ and $X$ the linearized branch flow model can be summarized compactly as:

$$
v=\bar{v}_{0}+R\left(p^{g}-p^{c}\right)+X\left(q^{g}-q^{c}\right),
$$

where $\bar{v}_{0}=\left[v_{0}, \ldots, v_{0}\right]^{\top}$ is an $n$-dimensional vector. In this paper we assume that $\bar{v}_{0}, p^{g}, p^{c}, q^{c}$ are given constants. The only variables are (column) vectors $v:=\left[v_{1}, \ldots, v_{n}\right]^{\top}$ of squared voltage magnitudes and $q^{g}:=\left[q_{1}^{g}, \ldots, q_{n}^{g}\right]$ of generated reactive powers. Let $\tilde{v}=\bar{v}_{0}+R\left(p^{g}-p^{c}\right)-X q^{c}$, which is a constant vector. For notational simplicity, we will henceforth ignore the superscript in $q^{g}$ and write $q$ instead. Then the linearized branch flow model reduces to the following simple form:

$$
v=X q+\tilde{v} .
$$

We have the following result.

Lemma 1 The matrices $R$ and $X$ are positive definite. 
Proof: The proof uses the fact that the resistance and reactance values of power lines in the network are all positive. ${ }^{1}$ Here the same argument applies to the resistance matrix $R$.

We prove by induction on the number $k$ of buses in the network, excluding bus 0 (the root bus). The base case of $k=1$ corresponds to a two-bus network with one line. Here $X$ is a positive scalar that is equal to the reactance of the line connecting the two buses.

Suppose that the theorem holds for all $k \leq n$. For the case of $k=n+1$ we consider two possible network topologies as shown in Figure 2:

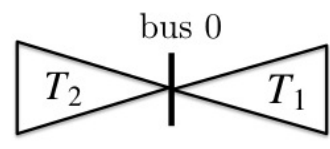

(a) Case 1: degree of bus 0 is greater than 1 .

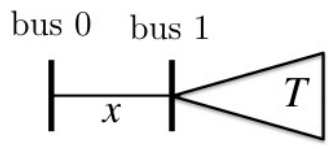

(b) Case 2: degree of bus 0 is
Fig. 2. Two possible network structures.

Case 1: bus 0 is of degree greater than 1. Split the network into two different trees rooted at bus 0 , denoted by $T_{1}$ and $T_{2}$, each of which has no more than $n$ buses excluding bus 0 . Denote by $X_{1}$ and $X_{2}$ respectively the reactance matrices of $\mathcal{T}_{1}$ and $\mathcal{T}_{2}$. By induction assumption $X_{1}$ and $X_{2}$ are positive definite. Note that the set $\mathcal{L}_{i}$ of lines on the unique path from bus 0 to bus $i$ must completely lie inside either $\mathcal{T}_{1}$ or $\mathcal{T}_{2}$, for all $i$. Therefore, by definition (2), the reactance matrix $X$ of the network has the following block-diagonal form:

$$
X_{i j}=\left\{\begin{array}{cl}
X_{1_{i j}}, & i, j \in \mathcal{T}_{1} \\
X_{2_{i j}}, & i, j \in \mathcal{T}_{2} \\
0, & \text { otherwise }
\end{array} \quad \Rightarrow X=\left[\begin{array}{cc}
X_{1} & 0 \\
0 & X_{2}
\end{array}\right] .\right.
$$

Since $X_{1}$ and $X_{2}$ are positive definite, so is $X$.

Case 2: bus 0 is of degree 1. Suppose without loss of generality that bus 0 is connected to bus 1 . Denote by $x$ the reactance of the line connecting buses 0 and 1 , and $\mathcal{T}$ the tree rooted at bus 1, excluding bus 0 . Denote by $Y$ the reactance matrix of $\mathcal{T}$, and by induction assumption, $Y$ is positive definite. Note that, for all buses $i$ in the network, the set $\mathcal{L}_{i}$ includes the single line that connects buses 0 and 1. Therefore, by definition (2), the reactance matrix X has the following form:

$X_{i j}=\left\{\begin{array}{cc}Y_{i j}+x, \quad i, j \in \mathcal{T} \\ x, & \text { otherwise }\end{array} \Rightarrow X=\left[\begin{array}{cc}x & \ldots x \\ \vdots & \vdots \\ x & \ldots x\end{array}\right]+\left[\begin{array}{cc}0 & 0 \\ 0 & Y\end{array}\right]\right.$,

One can verify that, when $Y$ is positive definite and $x$ is positive, $X$ is positive definite. This concludes the proof.

We also refer to [26] for an alternative proof for the same result.

\footnotetext{
${ }^{1}$ This is true for both over-head and underground power lines. The underground lines usually have larger capacitances and thus smaller reactances than the over-head lines. But their reactances are still positive because of the dominance of inductance; see, e.g., Table 3.1 in [46].

Nonetheless, there may be situations where capacitance dominates inductance such that the overall reactance is negative. In view of this, we limit the applicability of Lemma 1 and other results of this paper to power networks where reactances are positive.
}

\section{Inverter Model}

At each bus $i$ there is an inverter that can generate nonnegative real power $p_{i}$ and reactive power $q_{i}$ that can have either sign. $p_{i}$ and $q_{i}$ are constrained by the apparent power capability $s_{i}$ of the inverter as follows:

$$
0 \leq p_{i} \leq s_{i}, \quad 0 \leq\left|q_{i}\right| \leq s_{i}, \quad p_{i}^{2}+q_{i}^{2} \leq s_{i}^{2} .
$$

Consider power ratio $\cos \rho_{i}$ with $0 \leq \rho_{i} \leq \pi / 2$ such that

$$
p_{i} / s_{i} \geq \cos \rho_{i} .
$$

Given non-controllable $p_{i} \leq s_{i}$, the feasible (reactive) power set $\Omega_{i}$ for inverter $i$ is given by:

$$
\Omega_{i}:=\left\{q_{i} \mid q_{i}^{\min } \leq q_{i} \leq q_{i}^{\max }\right\},
$$

where, based on (6)-(7),

$$
\begin{aligned}
q_{i}^{\max } & =\min \left\{p_{i} \tan \rho_{i}, \sqrt{s_{i}^{2}-p_{i}^{2}}\right\}, \\
q_{i}^{\min } & =\max \left\{-p_{i} \tan \rho_{i},-\sqrt{s_{i}^{2}-p_{i}^{2}}\right\} .
\end{aligned}
$$

Here, $p_{i}$ is further assumed to be sized appropriately to provide enough freedom in $q_{i}$ [31]. For buses without controllable inverters, we can set $q_{i}=q_{i}^{\max }=q_{i}^{\min }$ to make $\Omega_{i}$ a singleton. Define $\Omega:=\mathrm{X}_{i=1}^{n} \Omega_{i}$ for notational simplicity.

\section{Local Volt/VAR Control}

The goal of Volt/VAR control in a distribution network is to maintain the bus voltages $v$ to within a tight range around their nominal values $v_{i}^{\text {nom }}=1$ p.u., $i \in \mathcal{N}$ by provisioning reactive power injections $q$. This can be modeled as a feedback dynamical system with state $(v(t), q(t))$ at discrete time $t$. A general Volt/VAR control algorithm maps the current state $(v(t), q(t))$ to a new reactive power injections $q(t+1)$. The new $q(t+1)$ updates voltage magnitudes $v(t+1)$ according to (5). Usually $q(t+1)$ is determined either completely or partly by a certain Volt/VAR control function defined as follows:

Definition 1 A Volt/VAR control function $f: \mathbb{R}^{n} \rightarrow \mathbb{R}^{n}$ is a collection of local control functions $f_{i}: \mathbb{R} \rightarrow \mathbb{R}$, each of which maps the current local voltage $v_{i}$ to a local control variable $u_{i}$ in reactive power at bus $i$ :

$$
u_{i}=f_{i}\left(v_{i}-v_{i}^{n o m}\right) . \quad \forall i \in \mathcal{N} .
$$

The control functions $f_{i}$ are usually decreasing but not always strictly decreasing because of a potential deadband where the control signal $u_{i}$ is set to zero to prevent too frequent actuation. We assume for each bus $i \in \mathcal{N}$ a symmetric deadband $\left(v_{i}^{\text {nom }}-\delta_{i} / 2, v_{i}^{\text {nom }}+\delta_{i} / 2\right)$ with $\delta_{i} \geq 0$ around the nominal voltage $v_{i}^{\text {nom }}$. The following assumptions are made for $f_{i}$.

Assumption 1 The control functions $f_{i}$ are continuous, nonincreasing in $\mathbb{R}$, and strictly decreasing and differentiable in $\left(-\infty,-\delta_{i} / 2\right) \cup\left(\delta_{i} / 2,+\infty\right)$.

Assumption 2 The derivative of $f_{i}$ is upper-bounded, i.e., there exist $\bar{\alpha}_{i}>0$ such that $\left|f_{i}^{\prime}\left(v_{i}\right)\right| \leq \bar{\alpha}_{i}$ for all feasible $v_{i} \in\left(-\infty,-\delta_{i} / 2\right) \cup\left(-\delta_{i} / 2, \delta_{i} / 2\right) \cup\left(\delta_{i} / 2,+\infty\right), \forall i \in \mathcal{N}$. 
Assumption 2 ensures that an infinitesimal change in voltage does not lead to a jump in the control variable. Define $\bar{A}:=$ $\operatorname{diag}\left\{\bar{\alpha}_{1}, \ldots, \bar{\alpha}_{n}\right\} \in \mathbb{S}_{++}^{N}$, and let $M=\sigma_{\max }(\bar{A} X)$ denote the largest singular value of $\bar{A} X$. We have the following result.

Lemma 2 (Lipschitz continuity) Suppose Assumptions 1-2 hold. For any $q, q^{\prime} \in \Omega$, we have

$$
\left\|f\left(v(q)-v^{n o m}\right)-f\left(v\left(q^{\prime}\right)-v^{n o m}\right)\right\|_{2} \leq M\left\|q-q^{\prime}\right\|_{2} .
$$

Proof: Without loss of generality, assume that $v_{i}(q) \geq$ $v_{i}\left(q^{\prime}\right)$. If both $v_{i}(q)$ and $v_{i}\left(q^{\prime}\right)$ are in $\left(-\infty, v_{i}^{\text {nom }}-\delta_{i} / 2\right]$ or in $\left[v_{i}^{\text {nom }}+\delta_{i} / 2,+\infty\right)$, by the mean value theorem we have $\left|f_{i}\left(v_{i}(q)-v_{i}^{\text {nom }}\right)-f_{i}\left(v_{i}\left(q^{\prime}\right)-v_{i}^{\text {nom }}\right)\right| \leq \bar{\alpha}_{i}\left|v_{i}(q)-v_{i}\left(q^{\prime}\right)\right|$. If both are in $\left[v_{i}^{\text {nom }}-\delta_{i} / 2, v_{i}^{\text {nom }}+\delta_{i} / 2\right], 0=\mid f_{i}\left(v_{i}(q)-v_{i}^{\text {nom }}\right)-f_{i}\left(v_{i}\left(q^{\prime}\right)-\right.$ $\left.v_{i}^{\text {nom }}\right)\left|\leq \bar{\alpha}_{i}\right| v_{i}(q)-v_{i}\left(q^{\prime}\right) \mid$. If $v_{i}(q) \in\left[v_{i}^{\text {nom }}+\delta_{i} / 2,+\infty\right)$ and $v_{i}\left(q^{\prime}\right) \in\left[v_{i}^{\text {nom }}-\delta_{i} / 2, v_{i}^{\text {nom }}+\delta_{i} / 2\right], \mid f_{i}\left(v_{i}(q)-v_{i}^{\text {nom }}\right)-f_{i}\left(v_{i}\left(q^{\prime}\right)-\right.$ $\left.\left.v_{i}^{\text {nom }}\right)|=| f_{i}\left(v_{i}(q)-v_{i}^{\text {nom }}\right)-f_{i}\left(\delta_{i} / 2\right)\right)\left|\leq \bar{\alpha}_{i}\right| v_{i}(q)-\left(v_{i}^{\text {nom }}+\right.$ $\left.\delta_{i} / 2\right)\left|\leq \bar{\alpha}_{i}\right| v_{i}(q)-v_{i}\left(q^{\prime}\right) \mid$, where the first inequality follows from the mean value theorem. Similarly, we can show that $\left|f_{i}\left(v_{i}(q)-v_{i}^{\text {nom }}\right)-f_{i}\left(v_{i}\left(q^{\prime}\right)-v_{i}^{\text {nom }}\right)\right| \leq \bar{\alpha}_{i}\left|v_{i}(q)-v_{i}\left(q^{\prime}\right)\right|$ holds under other situations too. Therefore, the following holds:

$$
\left\|f\left(v(q)-v^{\mathrm{nom}}\right)-f\left(v\left(q^{\prime}\right)-v^{\mathrm{nom}}\right)\right\|_{2} \leq\left\|\bar{A}\left(v(q)-v\left(q^{\prime}\right)\right)\right\|_{2} .
$$

Then by the definition of $M$ we have (10).

See Fig. 3 (left) for an illustrative example of a piecewise linear droop control function based on IEEE Standard 1547 [5]:

$$
f_{i}\left(v_{i}\right)=-\alpha_{i}\left[v_{i}-\delta_{i} / 2\right]^{+}+\alpha_{i}\left[-v_{i}-\delta_{i} / 2\right]^{+}
$$

with slope $-\alpha_{i}$ in $\left(-\infty,-\delta_{i} / 2\right)$ and $\left(\delta_{i} / 2,+\infty\right)$. Notice that our design and analysis in this paper are not limited to linear control functions.

Motivated by the IEEE Standard 1547, we first consider a "non-incremental" control where the reactive power $q_{i}=$ $u_{i}, i \in \mathcal{N}$, and obtain the following dynamical system $\mathcal{D}_{1}$ for the local Volt/VAR control:

$$
\mathcal{D}_{1}:\left\{\begin{aligned}
v(t) & =X q(t)+\tilde{v} \\
q_{i}(t+1) & =\left[f_{i}\left(v_{i}(t)-v_{i}^{\text {nom }}\right)\right]_{\Omega_{i}}, i \in \mathcal{N},
\end{aligned}\right.
$$

where [ $]_{\Omega_{i}}$ denotes the projection onto the set $\Omega_{i}$. A fixed point $\left(v^{*}, q^{*}\right)$ of the above dynamical system, defined as follows, represents an equilibrium operating point of the network.

Definition $2\left(v^{*}, q^{*}\right)$ is called an equilibrium point of $\mathcal{D}_{1}$, if it satisfies

$$
\begin{aligned}
v^{*} & =X q^{*}+\tilde{v}, \\
q^{*} & =\left[f\left(v^{*}-v^{n o m}\right)\right]_{\Omega} .
\end{aligned}
$$

In the next section, we characterize the equilibrium and dynamical properties of the system $\mathcal{D}_{1}$ by showing that it is an distributed algorithm for solving a well-defined optimization problem.

\section{ReVERSE ENGINEERING}

Since $f_{i}$ is non-increasing, a (generalized) inverse $f_{i}^{-1}$ exists. In particular, at the origin, we assign $f_{i}^{-1}(0)=0$ corresponding to the deadband $\left[-\delta_{i} / 2,+\delta_{i} / 2\right]$ of $f_{i}$. This may introduce discontinuity to $f_{i}^{-1}$ at $q_{i}=0$ if the deadband $\delta_{i}>0$, i.e.,

$$
f_{i}^{-1}\left(0^{+}\right) \leq-\delta_{i} / 2 \text { and } f_{i}^{-1}\left(0^{-}\right) \geq \delta_{i} / 2,
$$

where $0^{+}$and $0^{-}$represent approaching 0 from right and left, respectively.

Define a cost function for provisioning reactive power at each bus $i \in \mathcal{N}$ as:

$$
C_{i}\left(q_{i}\right):=-\int_{0}^{q_{i}} f_{i}^{-1}(q) d q
$$

which is convex since $f_{i}^{-1}$ is decreasing. Then, given $v_{i}(t)$, $q_{i}(t+1)$ in $(12 \mathrm{~b})$ is the unique solution to the following optimization problem:

$$
q_{i}(t+1)=\underset{q_{i} \in \Omega_{i}}{\arg \min } C_{i}\left(q_{i}\right)+q_{i}\left(v_{i}(t)-v_{i}^{\mathrm{nom}}\right),
$$

i.e., (12b) and (16) are equivalent specification of $q_{i}(t+1)$.

Take the piecewise linear control function (11) for example. Its inverse is given by:

$$
f_{i}^{-1}\left(q_{i}\right):= \begin{cases}-\frac{q_{i}}{\alpha_{i}}+\frac{\delta_{i}}{2}, & \text { if } q_{i}<0 \\ 0, & \text { if } q_{i}=0 \\ -\frac{q_{i}}{\alpha_{i}}-\frac{\delta_{i}}{2}, & \text { if } q_{i}>0\end{cases}
$$

and the corresponding cost function is given by:

$$
C_{i}\left(q_{i}\right)= \begin{cases}\frac{1}{2 \alpha_{i}} q_{i}^{2}-\frac{\delta_{i}}{2} q_{i}, & \text { if } q_{i} \leq 0, \\ \frac{1}{2 \alpha_{i}} q_{i}^{2}+\frac{\delta_{i}}{2} q_{i}, & \text { if } q_{i}>0 .\end{cases}
$$

See Fig. 3 (middle and right) for illustration.

\section{A. Equilibrium}

Consider the function $F(q): \Omega \rightarrow \mathbb{R}$ :

$$
F(q):=C(q)+\frac{1}{2} q^{\top} X q+q^{\top} \Delta \tilde{v},
$$

where $C(q)=\sum_{i \in \mathcal{N}} C_{i}\left(q_{i}\right)$ and $\Delta \tilde{v}:=\tilde{v}-v^{\text {nom }}$, and a global optimization problem:

$$
\min _{q \in \Omega} F(q) .
$$

Theorem 1 Suppose Assumption 1 holds. Then $\mathcal{D}_{1}$ has a unique equilibrium point. Moreover, a point $\left(v^{*}, q^{*}\right)$ is an equilibrium of $\mathcal{D}_{1}$ if and only if $q^{*}$ is the unique optimal solution of (20) and $v^{*}=X q^{*}+\tilde{v}$.

Proof: By Lemma 1 the matrix $X$ is positive definite. This implies that the objective function $F(q)$ is strongly convex. Hence, the first-order optimality condition for (20) is both necessary and sufficient; moreover, (20) has a unique optimal solution. We next relate it to the equilibrium point of $\mathcal{D}_{1}$.

The subdifferential of $F(q)$ is given by:

$$
\begin{aligned}
\partial F(q) & =\partial C(q)+X q+\Delta \tilde{v} \\
& =\partial C(q)+(X q+\tilde{v})-v^{\mathrm{nom}},
\end{aligned}
$$

where, by the definition of $C_{i}\left(q_{i}\right)$,

$$
\partial C(q)=\left[\partial C_{1}\left(q_{1}\right), \ldots, \partial C_{n}\left(q_{n}\right)\right]^{\top}
$$




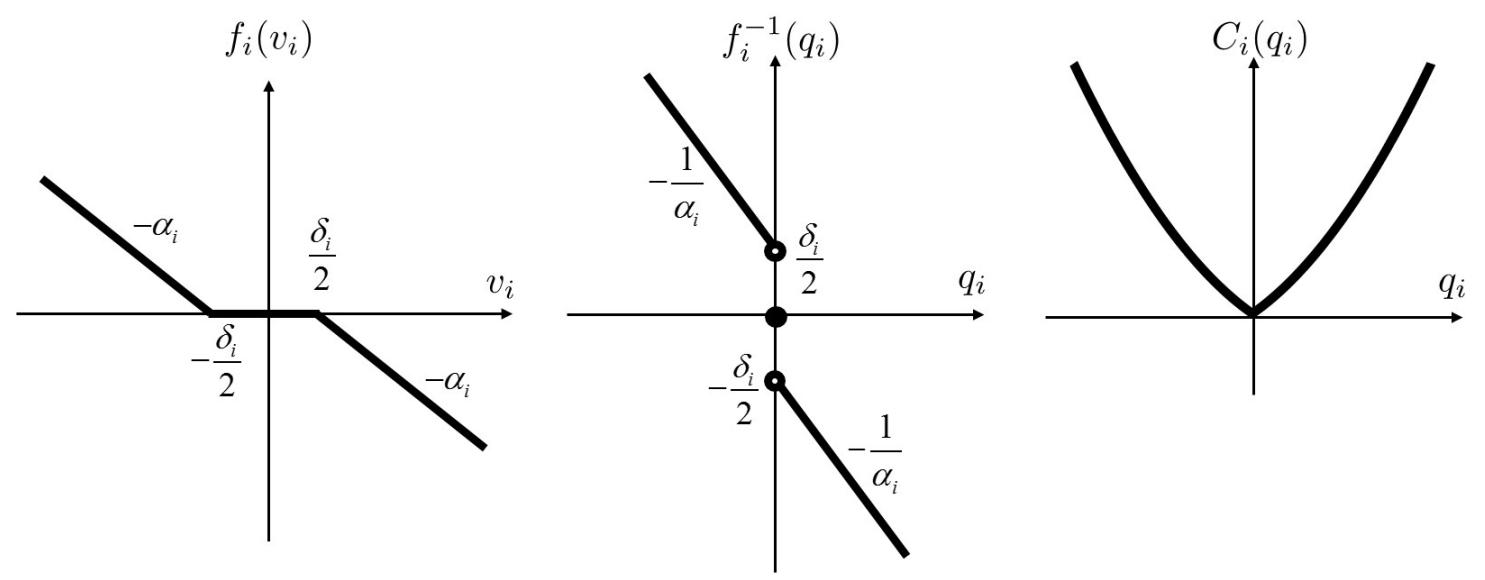

Fig. 3. (left) The piecewise linear control function (11), (middle) its inverse function (17), and (right) the corresponding cost function (18).

with

$$
\partial C_{i}\left(q_{i}\right)= \begin{cases}-f_{i}^{-1}\left(q_{i}\right), & \text { if } q_{i} \neq 0 \\ {\left[-\frac{\delta_{i}}{2}, \frac{\delta_{i}}{2}\right],} & \text { if } q_{i}=0\end{cases}
$$

By the optimality condition, $q^{*}$ is an optimum of (20) iff there exists a (sub)gradient $\nabla F\left(q^{*}\right) \in \partial F\left(q^{*}\right)$ such that

$$
\nabla F\left(q^{*}\right)^{\top}\left(q-q^{*}\right) \geq 0, \forall q \in \Omega,
$$

which is equivalent to:

$$
q^{*}=\left[f\left(X q^{*}+\tilde{v}-v^{\mathrm{nom}}\right)\right]_{\Omega} .
$$

It follows that a point $\left(v^{*}, q^{*}\right)$ is an equilibrium of $\mathcal{D}_{1}$ if and only if $q^{*}$ solves (20) and $v^{*}=X q^{*}+\tilde{v}$. The existence and uniqueness of the optimal solution of (20) then implies that of the equilibrium $\left(v^{*}, q^{*}\right)$.

By $v=X q+\tilde{v}$, the objective can be equivalently written as:

$F(q, v)=C(q)+\frac{1}{2}\left(v-v^{\mathrm{nom}}\right)^{\top} X^{-1}\left(v-v^{\mathrm{nom}}\right)-\frac{1}{2} \Delta \tilde{v}^{\top} X^{-1} \Delta \tilde{v}$.

Notice that the last term is a constant. Therefore, the local Volt/VAR control $\mathcal{D}_{1}$ seeks an optimal trade-off between minimizing the cost of reactive power provisioning $C(q)$ and minimizing the cost of voltage deviation $\frac{1}{2}\left(v-v^{\text {nom }}\right)^{\top} X^{-1}\left(v-v^{\text {nom }}\right)$.

1) Further Characterization of Equilibrium: The first term $C(q)$ of the objective (21) is well-defined and has the desired additive structure. It is however not clear what specific structure the second term $\frac{1}{2}\left(v-v^{\text {nom }}\right)^{\top} X^{-1}\left(v-v^{\text {nom }}\right)$ entails. We further characterize this term in this subsection.

Notice that bus 0 has a fixed voltage magnitude, which decouples different subtrees rooted at it. Therefore without loss of generality, we only consider a topology where the bus 0 is of degree 1 . Denote $\mathcal{T}$ the (sub)tree rooted at bus 1 and $\mathcal{L}_{T}$ the set of links of $\mathcal{T}$. Define an inverse tree $\mathcal{T}^{\prime}$ that has the same sets of buses and lines as $\mathcal{T}$ but with reciprocal line reactance $1 / x_{i j}$. Let $\mathbb{L} \in \mathbb{R}^{n \times n}$ be the weighted Laplacian matrix of $\mathcal{T}^{\prime}$ defined as follows:

$$
\mathbb{L}_{i j}= \begin{cases}-1 / x_{i j}, & (i, j) \in \mathcal{L}_{T}, \\ \sum_{(i, k) \in \mathcal{L}} 1 / x_{i k}, & i=j, \\ 0, & \text { otherwise. }\end{cases}
$$

Recall that $x$ denotes the reactance of the line connecting buses 0 and 1, we have the following result by Liu et al. [47].

Theorem 2 (Lemma 6 in [47]) Given the tree graph $\mathcal{G}=$ $\{\mathcal{N} \cup\{0\}, \mathcal{L}\}$ with bus 0 being of degree 1 and its reactance matrix $X$ defined by (2), the inverse matrix $X^{-1}$ has the following explicit form:

$$
X^{-1}=\mathbb{L}+\left[\begin{array}{cccc}
1 / x & 0 & \cdots & 0 \\
0 & 0 & \cdots & 0 \\
\vdots & \vdots & \ddots & \vdots \\
0 & 0 & \cdots & 0
\end{array}\right] .
$$

With the above result, the cost function (21) can be rewritten as:

$$
\begin{gathered}
F(q, v)=C(q)+\frac{1}{2}\left(\frac{\left(v_{1}-v^{\mathrm{nom}}\right)^{2}}{x}+\sum_{(i, j) \in \mathcal{L}_{T}} \frac{\left(v_{i}-v_{j}\right)^{2}}{x_{i j}}\right) \\
-\frac{1}{2} \Delta \tilde{v}^{\top} X^{-1} \Delta \tilde{v} .
\end{gathered}
$$

whose second term (i.e., the cost of voltage deviation) consists of two parts: the first part $\left(v_{1}-v^{\text {nom }}\right)^{2} / x$ represents the cost of voltage deviation of the bus 1 from the nominal value, and the second part $\sum_{(i, j) \in \mathcal{L}_{T}}\left(v_{i}-v_{j}\right)^{2} / x_{i j}$ gives the cost of voltage deviation between the neighboring buses. This leads to a nice leader-follower structure where the first bus (the bus 1) aims to attain the nominal voltage while every other bus tries to achieve the same voltage as that of the bus "in front of" it.

\section{B. Dynamics}

We now study the dynamical properties of the local Volt/VAR control $\mathcal{D}_{1}$.

Theorem 3 Suppose Assumptions 1-2 hold. If

$$
\sigma_{\max }(\bar{A} X)<1
$$

then the local Volt/VAR control $\mathcal{D}_{1}$ converges to the unique equilibrium point $\left(v^{*}, q^{*}\right)$. Moreover, it converges exponentially fast to the equilibrium. 
Proof: Write $\mathcal{D}_{1}$ equivalently as a mapping $g_{1}: \Omega \rightarrow \Omega$ :

$$
q(t+1)=g_{1}(q(t)):=\left[f\left(X q(t)+\Delta \tilde{v}-v^{\text {nom }}\right)\right]_{\Omega} .
$$

By Lemma 2 and the non-expansiveness property of projection operator, given any feasible $q, q^{\prime}$ we have

$$
\left\|g_{1}(q)-g_{1}\left(q^{\prime}\right)\right\|_{2} \leq M\left\|q-q^{\prime}\right\|_{2},
$$

where $M=\sigma_{\max }(\bar{A} X)$. When condition (24) holds, $M<1$ and thus the mapping $g_{1}$ is a contraction, implying that $(v(t), q(t))$ converges exponentially to the unique equilibrium point under $\mathcal{D}_{1}$.

We next develop a sufficient condition for (24), which is easier to verify in practice. Define the following matrix norms for some $W \in \mathbb{R}^{m \times n}$ :

$$
\begin{aligned}
& \|W\|_{1}=\max _{1 \leq j \leq n} \sum_{i=1}^{m}\left|w_{i j}\right|, \quad\|W\|_{\infty}=\max _{1 \leq i \leq m} \sum_{j=1}^{n}\left|w_{i j}\right|, \\
& \|W\|_{2}=\sqrt{\lambda_{\max }\left(W^{\top} W\right)}=\sigma_{\max }(W),
\end{aligned}
$$

where $\lambda_{\max }(\cdot)$ denotes the largest eigenvalue of a matrix. By utilizing the following relationship among these matrix norms based on Hölder's inequality

$$
\|W\|_{2} \leq \sqrt{\|W\|_{1} \cdot\|W\|_{\infty}},
$$

we have the following sufficient condition for convergence of $\mathcal{D}_{1}$.

Corollary 1 Suppose Assumptions 1-2 hold. If

$$
\max _{i \in \mathcal{N}}\left(\bar{\alpha}_{i}\right) \cdot \max _{i \in \mathcal{N}}\left(\sum_{j \in \mathcal{N}} X_{i j}\right)<1,
$$

then $\mathcal{D}_{1}$ converges exponentially fast to the unique equilibrium point $\left(v^{*}, q^{*}\right)$.

Proof: A sufficient condition for (24) based on (27) is

$$
\|\bar{A} X\|_{1}<1 \text { and }\|\bar{A} X\|_{\infty}<1 .
$$

Therefore given symmetric matrix $X,(28)$ is sufficient for (29).

\section{Limitation of the Non-Incremental Control}

The local voltage control (12b) is non-incremental, as it decides the total amount of reactive power (instead of the change in reactive power) based on the deviation of the current voltage from the nominal value. Intuitively, such control may lead to over-actuation and oscillatory behavior. In order to have converging or stable behavior, the control function should not be too aggressive, i.e., have a small (absolute) derivative. This can also be seen from Theorem 3, and in the case of the piecewise linear control function (11), implies a small $\alpha_{i}$ value.

On the other hand, seen from the equivalent objective (21), smaller cost functions $C_{i}\left(q_{i}\right)$ are preferred for better voltage regulation. However, a small cost function implies large derivative of the control function; see, e.g., the cost function (18) that becomes smaller as $\alpha_{i}$ takes larger value, as well as the numerical examples in Section V.

Hence, there is a contention or fundamental limitation for the non-incremental control: control function with smaller derivative is preferred for convergence, while for better voltage regulation at the equilibrium control function with larger derivative is desired. This motivates us to seek new local voltage control schemes that are not subject to such a limitation, as will be seen in the next section.

\section{Forward Engineering: Decoupling Equilibrium and Dynamical Properties}

The optimization-based model (20) not only provides a way to characterize the equilibrium of the local voltage control (see Theorem 1) but also suggests a principled way to engineer the control. New design goal such as fairness and economic efficiency can be incorporated by engineering the objective function in (20), and more importantly, new control schemes with better dynamical properties can be designed based on various optimization algorithms such as a (sub)gradient algorithm. In this section, we apply two iterative optimization algorithms to design local voltage control schemes that can decouple the dynamical property from the equilibrium property and have less restrictive convergence conditions than the non-incremental local voltage control studied in the previous section.

\section{A. Local Voltage Control Based on (Sub)gradient Algorithm}

Given an optimization problem, we may apply different algorithms to solve it. A common algorithm that often admits distributed implementation is the (sub)gradient method [48]. Applying it to the problem (20) leads to the following voltage control:

$$
q_{i}(t+1)=\left[q_{i}(t)-\gamma_{2} \frac{\partial F(q(t))}{\partial q_{i}}\right]_{\Omega_{i}}, i \in \mathcal{N},
$$

where $\gamma_{2}>0$ is a constant stepsize and the (sub)gradient is calculated as follows:

$$
\frac{\partial F(q(t))}{\partial q_{i}}=\left\{\begin{array}{lr}
-f_{i}^{-1}\left(q_{i}(t)\right)+v_{i}(t)-v^{\mathrm{nom}}, & \text { if } q_{i}(t) \neq 0, \\
v_{i}(t)-v^{\mathrm{nom}}, & \text { if } q_{i}(t)=0 \text { and } \\
& -\delta / 2 \leq v_{i}(t)-v^{\mathrm{nom}} \leq \delta_{i} / 2, \\
-\delta_{i} / 2+v_{i}(t)-v^{\mathrm{nom}}, & \text { if } q_{i}(t)=0 \text { and } \\
& v_{i}(t)-v^{\mathrm{nom}}>\delta_{i} / 2, \\
& \text { if } q_{i}(t)=0 \text { and } \\
\delta_{i} / 2+v_{i}(t)-v^{\mathrm{nom}}, & v_{i}(t)-v^{\mathrm{nom}}<-\delta_{i} / 2 .
\end{array}\right.
$$

The above control is incremental as the change in reactive power (instead of the total reactive power) is based on the voltage deviation from the nominal value. It is also distributed since the decision at each bus $i \in \mathcal{N}$ depends only on its current reactive power $q_{i}$ and voltage $v_{i}$.

We thus obtain the following dynamical system

$$
\mathcal{D}_{2}:\left\{\begin{aligned}
v(t) & =X q(t)+\tilde{v} \\
q_{i}(t+1) & =\left[q_{i}(t)-\gamma_{2} \frac{\partial F(q(t))}{\partial q_{i}}\right]_{\Omega_{i}}, i \in \mathcal{N} .
\end{aligned}\right.
$$

The following result is immediate.

Theorem 4 Suppose Assumption 1 holds. Then there exists a unique equilibrium point for the dynamical system $\mathcal{D}_{2}$. 
Moreover, a point $\left(v^{*}, q^{*}\right)$ is an equilibrium if and only if $q^{*}$ is the unique optimal solution of problem (20) and $v^{*}=X q^{*}+\tilde{v}$.

Proof: The proof is similar to that for Theorem 1. At the equilibrium $q^{*}$ of dynamical system $\mathcal{D}_{2}$, we have

$$
q_{i}^{*}=\left[q_{i}^{*}-\gamma_{2} \frac{\partial F\left(q^{*}\right)}{\partial q_{i}}\right]_{\Omega_{i}}, i \in \mathcal{N},
$$

which is equivalent to

$$
\frac{\partial F\left(q^{*}\right)}{\partial q_{i}}\left(\bar{q}_{i}-q_{i}^{*}\right) \geq 0, \quad \forall \bar{q}_{i} \in \Omega_{i},
$$

i.e., $\nabla F\left(q^{*}\right)^{T}\left(\bar{q}-q^{*}\right) \geq 0, \quad \forall \bar{q} \in \Omega$. So, $q^{*}$ satisfies the optimality condition and thus is an optimum of problem (20). The uniqueness of equilibrium/optimum comes from the fact that convex problem (20) has a strongly convex objective function.

Since the feasible sets are bounded, we also have the bounded (sub)gradient of $F(q)$ with some constant $G>0$ :

$$
\left\|\nabla_{q} F(q)\right\|_{2} \leq G, \forall q \in \Omega .
$$

Theorem 5 Suppose Assumption 1 holds. The dynamical system $\mathcal{D}_{2}$ converges as

$$
\limsup _{t \rightarrow \infty} \sum_{\tau=1}^{t} \frac{F(q(\tau))-F\left(q^{*}\right)}{t}=\gamma_{2}^{2} G^{2} .
$$

Proof: We characterize the distance between $q(t+1)$ and $q^{*}$ as:

$$
\begin{aligned}
& \left\|q(t+1)-q^{*}\right\|_{2}^{2} \\
\leq & \left\|q(t)-\gamma_{2} \nabla_{q} F(q(t))-q^{*}\right\|_{2}^{2} \\
= & \left\|q(t)-q^{*}\right\|_{2}^{2}+\gamma_{2}^{2}\left\|\nabla_{q} F(q(t))\right\|_{2}^{2}-2 \gamma_{2}\left(q(t)-q^{*}\right)^{\top} \nabla_{q} F(q(t)) \\
\leq & \left\|q(t)-q^{*}\right\|_{2}^{2}+\gamma_{2}^{2} G^{2}-\left(F(q(t))-F\left(q^{*}\right)\right) \\
\leq & \left\|q(1)-q^{*}\right\|_{2}^{2}+t \gamma_{2}^{2} G^{2}-\sum_{\tau=1}^{t}\left(F(q(\tau))-F\left(q^{*}\right)\right),
\end{aligned}
$$

where the first inequality is due to non-expansiveness of projection operator, the second inequality is because of the definition of subgradient as well as the bounded gradient (33), and the last inequality is by repeating previous steps.

As $\left\|q(t+1)-q^{*}\right\|_{2}^{2} \geq 0$, it follows that:

$$
\sum_{\tau=1}^{t} \frac{F(q(\tau))-F\left(q^{*}\right)}{t} \leq\left\|q(1)-q^{*}\right\|_{2}^{2} / t+\gamma_{2}^{2} G^{2} .
$$

When $t \rightarrow \infty$, we have (34).

Theorem 5 states that for any control functions $f_{i}$ (that satisfies Assumptions 1-2), one can always find a small enough stepsize $\gamma_{2}$ such that $\mathcal{D}_{2}$ converges to a neighborhood of the $\left(v^{*}, q^{*}\right)$ of required accuracy on running average. Moreover, as shown in [54], when $q^{*}$ is not close to the non-differentiable point, $\mathcal{D}_{2}$ converges exactly to the optimum with small enough constant stepsizes. In contrast, the convergence condition (24) for the non-incremental voltage control $\mathcal{D}_{1}$ does constrain the allowable control functions $f_{i}$. Therefore, $\mathcal{D}_{2}$ permits better voltage regulation than $\mathcal{D}_{1}$; see the discussion at the end of Section III and simulation results in Fig. 5.
Nevertheless, the (sub)gradient nature of $\mathcal{D}_{2}$ may prevent it from converging to the exact optimal point. This could happen when the optimum is close to the non-differentiable point $\left(q^{*}=0\right.$ in this case) where the value of subgradient (31) changes abruptly if $\delta_{i} \neq 0$. Moreover, the (sub)gradient computation (31) requires computing the inverse of the control function $f_{i}$, which can be computationally expensive for general control functions, as well as tracking the value of $v_{i}$ with respect to deadband $\pm \delta_{i} / 2$. These limitations motivate us to design another incremental control scheme with better convergence and lower implementation complexity.

\section{B. Local Voltage Control Based on Pseudo-Gradient Algo- rithm}

The pseudo-gradient can provide a good search direction for an optimization problem without requiring the objective function to be differentiable; see, e.g., [49]. Applying it to the problem (20) leads to the following incremental voltage control at bus $i \in \mathcal{N}$ :

$$
\begin{aligned}
q_{i}(t+1) & =\left[q_{i}(t)-\gamma_{3}\left(q_{i}(t)-f_{i}\left(v_{i}(t)-v_{i}^{\mathrm{nom}}\right)\right)\right]_{\Omega_{i}} \\
& =\left[\left(1-\gamma_{3}\right) q_{i}(t)+\gamma_{3} f_{i}\left(v_{i}(t)-v_{i}^{\mathrm{nom}}\right)\right]_{\Omega_{i}},
\end{aligned}
$$

where $\gamma_{3}>0$ is a constant stepsize/weight and $q_{i}-f_{i}\left(v_{i}-v_{i}^{\text {nom }}\right)$ is the pseudo-gradient. The above control is distributed, and is simpler to implement than the control (30).

With (36) we obtain the following dynamical system

$$
\mathcal{D}_{3}:\left\{\begin{aligned}
& v(t)=X q(t)+\tilde{v}, \\
& q_{i}(t+1)=\left[q_{i}(t)-\gamma_{3}\left(q_{i}(t)-f_{i}\left(v_{i}(t)-v_{i}^{\text {nom }}\right)\right)\right]_{\Omega_{i}}, \\
& i \in \mathcal{N} .
\end{aligned}\right.
$$

Notice that $\mathcal{D}_{3}$ has the same equilibrium condition as the dynamical systems $\mathcal{D}_{1}$ and $\mathcal{D}_{2}$. The following result is immediate.

Theorem 6 Suppose Assumption 1 holds. There exists a unique equilibrium point for the dynamical system $\mathcal{D}_{3}$. Moreover, a point $\left(v^{*}, q^{*}\right)$ is an equilibrium if and only if $q^{*}$ is the unique optimal solution of problem (20) and $v^{*}=X q^{*}+\tilde{v}$.

We now analyze the convergence of the dynamical system $\mathcal{D}_{3}$. We first introduce the following useful results.

Denote by $\nabla_{v} f$ the diagonal matrix with each diagonal entry $\left(\nabla_{v} f\right)_{i i}$ representing the (sub)gradient of $f_{i}$ with respect to $v_{i}$ defined as:

$$
\left(\nabla_{v} f\right)_{i i} \begin{cases}=f_{i}^{\prime}\left(v_{i}\right), & \text { if } v_{i} \in\left(-\infty,-\delta_{i} / 2\right) \cup \\ \in\left[f_{i}^{\prime}\left(v_{i}^{-}\right), f_{i}^{\prime}\left(v_{i}^{+}\right)\right], & \text {if } v_{i}=-\delta_{i} / 2, \\ \in\left[f_{i}^{\prime}\left(v_{i}^{+}\right), f_{i}^{\prime}\left(v_{i}^{-}\right)\right], & \text {if } v_{i}=\delta_{i} / 2,\end{cases}
$$

which is bounded as $-\bar{\alpha}_{i} \leq\left(\nabla_{v} f\right)_{i i} \leq 0$ based on Assumptions $1-2$. Denote by $\lambda$ any eigenvalue of the matrix $\nabla_{v} f \cdot X$. Consider $\nabla_{v} f \cdot X$ 's similar matrix $X^{1 / 2} \cdot \nabla_{v} f \cdot X^{1 / 2}$, which is symmetric and negative semidefinite with real and nonpositive eigenvalues. Therefore, eigenvalues of the original asymmetric matrix $\nabla_{v} f \cdot X$ are also real and nonpositive. Similarly, all the eigenvalues of $\bar{A} X$ are real and positive. 
Theorem 7 Suppose Assumptions 1-2 hold. If the stepsize $\gamma_{3}$ satisfies the following condition:

$$
0<\gamma_{3}<2 /\left(1+\lambda_{\max }(\bar{A} X)\right)
$$

then the dynamical system $\mathcal{D}_{3}$ converges to its unique equilibrium point.

Proof: Write $\mathcal{D}_{3}$ equivalently as a mapping $g_{3}: \Omega \rightarrow \Omega$ :

$$
q(t+1)=g_{3}(q(t)):=\left[\left(1-\gamma_{3}\right) q(t)+\gamma_{3} f(v(q(t)))\right]_{\Omega},
$$

where $v(q(t))=X q(t)+\Delta \tilde{v}-v^{\text {nom }}$. The Jacobian matrix of $g_{3}$ without projection operator is computed as

$$
\nabla_{q} g_{3}=\left(1-\gamma_{3}\right) \mathcal{I}_{n}+\gamma_{3} \nabla_{v} f \cdot X
$$

where $\mathcal{I}_{n}$ is $n \times n$ identity matrix. On the other hand, when projection operator is active for some $q_{i}$, notice that the term $\left(1-\gamma_{3}\right) q_{i}(t)+\gamma_{3} f_{i}(v(q(t)))$ inside the projection operator is continuous, so the value of $q_{i}(t+1)$ does not change with respect to a small change of $q(t)$, and hence the $i$ th row of the resulting $\nabla_{q} g_{3}$ is all 0 . Therefore, by Gershgorin circle theorem [50], the magnitude of $\nabla_{q} g_{3}$ 's eigenvalue without active projection has a larger bound than that with projection. Therefore, it is sufficient to consider $g_{3}$ without the projection operator in this proof.

Denote by $z$ the eigenvector of matrix $\nabla_{v} f \cdot X$ corresponding to $\lambda$. By definition one has $\nabla_{v} f \cdot X \cdot z=\lambda z$. Therefore,

$$
\nabla_{q} g_{3} z=\left(1-\gamma_{3}+\gamma_{3} \lambda\right) z
$$

that is, the corresponding eigenvalue of $\nabla_{q} g_{3}$ with respect to $\lambda$ is $1-\gamma_{3}+\gamma_{3} \lambda$. To ensure that $g_{3}$ is stable, we must have [29], [51]:

$$
-1<1-\gamma_{3}+\gamma_{3} \lambda<1
$$

for any eigenvalue $\lambda$ of $\nabla_{v} f X$. (39) is sufficient for the lefthand side of (43) and the right-hand side always holds because $\lambda$ is nonpositive. This completes the proof.

We conclude that the dynamical system $\mathcal{D}_{3}$ based on the pseudo-gradient algorithm converges to the unique optimum with proper constant stepsize, while the dynamical system $\mathcal{D}_{2}$ based on the subgradient algorithm only guarantees convergence to within a small neighborhood of the optimum on running average with constant stepsize.

Remark 1 Notice that, when $\gamma_{3} \leq 1$ in, the control (36) has a nice interpretation of the new decision $q_{i}(t+1)$ being a (positively-)weighted sum of the decision $q_{i}(t)$ at the previous time and the local control $u_{i}(t)=f_{i}\left(v_{i}(t)-v_{i}^{\text {nom }}\right)$ in reactive power. Similar approaches in literature are also identified as exponentially weighted moving average (EWMA) control and delayed control, etc. However, here we do not require $\gamma_{3} \leq 1$ for $\mathcal{D}_{3}$ to converge, as long as condition (39) is satisfied.

\section{Control Function Design for Better Equilibrium Voltage Regulation}

As mentioned earlier, one motivation to consider the incremental local voltage control algorithms in the last two subsections is to decouple the convergence property from the equilibrium property, so that we can choose proper control

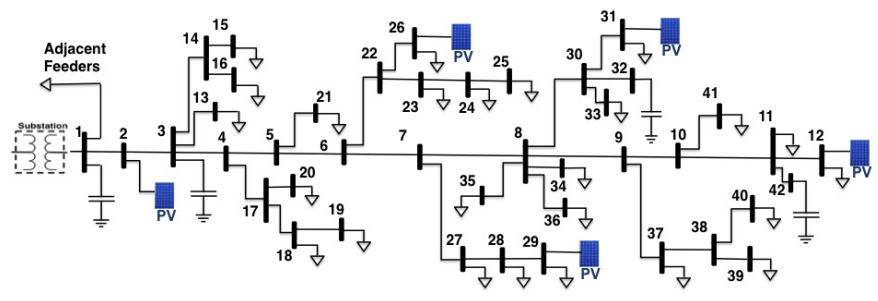

Fig. 4. Circuit diagram for SCE distribution system.

functions to achieve better equilibrium voltage profiles without risking the convergence of the system. In this subsection, we briefly discuss the design of control functions for better equilibrium voltage regulation.

Recall from Section III that, based on (21), local voltage control seeks an optimal trade-off between minimizing the cost of reactive power provisioning $\sum_{i} C_{i}\left(q_{i}\right)$ and minimizing the cost of voltage deviation $\frac{1}{2}\left(v-v^{\text {nom }}\right)^{\top} X^{-1}\left(v-v^{\text {nom }}\right)$. For better equilibrium voltage profile (i.e., closer to the nominal voltages), smaller cost functions $C_{i}\left(q_{i}\right)$ should be used, which by definition (15) means that control functions $f_{i}$ with larger (absolute) derivatives should be used; e.g., choose a large $\alpha_{i}$ value in the piecewise linear droop control function (11).

Notice that by choosing different control functions, we can achieve a "soft" control of equilibrium voltage profile. For the "hard" control or constraints of the voltage profile such as the voltage magnitude must fall into a specified range, we have to include these constraints explicitly in the optimization problem. There is work that considers the hard constraints and develops distributed voltage control algorithms, see, e.g., [14], [15], but it remains to be seen if local voltage control without any communication can achieve these hard constraints.

\section{Numerical EXAMPLes}

Consider a distribution feeder of South California Edison (SCE) with a high penetration of photovoltaic (PV) generation. As shown in Fig. 4, bus 1 is the substation (root bus) and five PV generators are integrated at buses 2, 12, 26, 29, and 31. As we aim to study the Volt/VAR control through PV inverters, all shunt capacitors are assumed to be off. Table I contains the network data including the line impedance, the peak MVA demand of loads, and the capacity of the PV generators. It is important to note that all studies are run with a full $\mathrm{AC}$ power flow model with MATPOWER [52] instead of its linear approximation. As will be seen, the results we develop for the linearized model are corroborated numerically with the full power flow model.

In all numerical studies, we implement the following homogeneous piecewise linear droop control functions of the IEEE 1547.8 Standard for all PV inverters with their deadbands from 0.98 p.u. to 1.02 p.u. and slopes $\alpha_{i}$ to be determined.

\section{A. Equilibrium}

As discussed in Section III-C, large (absolute) slopes of the control function lead to better voltage regulation at the equilibrium. To show this, we change $\alpha_{i}$ from 1 to 200 and 


\begin{tabular}{|c|c|c|c|c|c|c|c|c|c|c|c|c|c|c|c|c|c|}
\hline \multicolumn{18}{|c|}{ Network Data } \\
\hline \multicolumn{4}{|c|}{ Line Data } & \multicolumn{4}{|c|}{ Line Data } & \multicolumn{4}{|c|}{ Line Data } & \multicolumn{2}{|c|}{ Load Data } & \multicolumn{2}{|c|}{ Load Data } & \multicolumn{2}{|c|}{ PV Generators } \\
\hline From & To & $\mathrm{R}$ & $\mathrm{X}$ & From & To & $\mathrm{R}$ & $\mathrm{X}$ & From & To & $\mathrm{R}$ & $\mathrm{X}$ & Bus & Peak & Bus & Peak & Bus & Capacity \\
\hline Bus. & Bus. & $(\Omega)$ & $(\Omega)$ & Bus. & Bus. & $(\Omega)$ & $(\Omega)$ & Bus. & Bus. & $(\Omega)$ & $(\Omega)$ & No. & MVA & No. & MVA & No. & MW \\
\hline 1 & 2 & 0.259 & 0.808 & 8 & 34 & 0.244 & 0.046 & 18 & 19 & 0.198 & 0.046 & 11 & 0.67 & 28 & 0.27 & & \\
\hline 2 & 3 & 0.031 & 0.092 & 8 & 36 & 0.107 & 0.031 & 22 & 26 & 0.046 & 0.015 & 12 & 0.45 & 29 & 0.2 & 2 & 1 \\
\hline 3 & 4 & 0.046 & 0.092 & 8 & 30 & 0.076 & 0.015 & 22 & 23 & 0.107 & 0.031 & 13 & 0.89 & 31 & 0.27 & 12 & 3 \\
\hline 3 & 13 & 0.092 & 0.031 & 8 & 9 & 0.031 & 0.031 & 23 & 24 & 0.107 & 0.031 & 15 & 0.07 & 33 & 0.45 & 26 & 2 \\
\hline 3 & 14 & 0.214 & 0.046 & 9 & 10 & 0.015 & 0.015 & 24 & 25 & 0.061 & 0.015 & 16 & 0.67 & 34 & 1.34 & 29 & 1.8 \\
\hline 4 & 17 & 0.336 & 0.061 & 9 & 37 & 0.153 & 0.046 & 27 & 28 & 0.046 & 0.015 & 18 & 0.45 & 35 & 0.13 & 31 & 2.5 \\
\hline 4 & 5 & 0.107 & 0.183 & 10 & 11 & 0.107 & 0.076 & 28 & 29 & 0.031 & 0 & 19 & 1.23 & 36 & 0.67 & & \\
\hline 5 & 21 & 0.061 & 0.015 & 10 & 41 & 0.229 & 0.122 & 30 & 31 & 0.076 & 0.015 & 20 & 0.45 & 37 & 0.13 & & \\
\hline 5 & 6 & 0.015 & 0.031 & 11 & 42 & 0.031 & 0.015 & 30 & 32 & 0.076 & 0.046 & 21 & 0.2 & 39 & 0.45 & & \\
\hline 6 & 22 & 0.168 & 0.061 & 11 & 12 & 0.076 & 0.046 & 30 & 33 & 0.107 & 0.015 & 23 & 0.13 & 40 & 0.2 & & \\
\hline 6 & 7 & 0.031 & 0.046 & 14 & 16 & 0.046 & 0.015 & 37 & 38 & 0.061 & 0.015 & 24 & 0.13 & 41 & 0.45 & & \\
\hline 7 & 27 & 0.076 & 0.015 & 14 & 15 & 0.107 & 0.015 & 38 & 39 & 0.061 & 0.015 & 25 & 0.2 & & $V_{\text {base }}=$ & 2.35 & \\
\hline 7 & 8 & 0.015 & 0.015 & 17 & 18 & 0.122 & 0.092 & 38 & 40 & 0.061 & 0.015 & 26 & 0.07 & & $S_{\text {base }}=$ & 000 & \\
\hline 8 & 35 & 0.046 & 0.015 & 17 & 20 & 0.214 & 0.046 & & & & & 27 & 0.13 & & $Z_{\text {base }}=$ & 152.5 & \\
\hline
\end{tabular}

Network Parameters of the SCE Circuit: Line impedances, PEAK SPot load KVA, Capacitors and PV GENeration's namePlate Ratings.

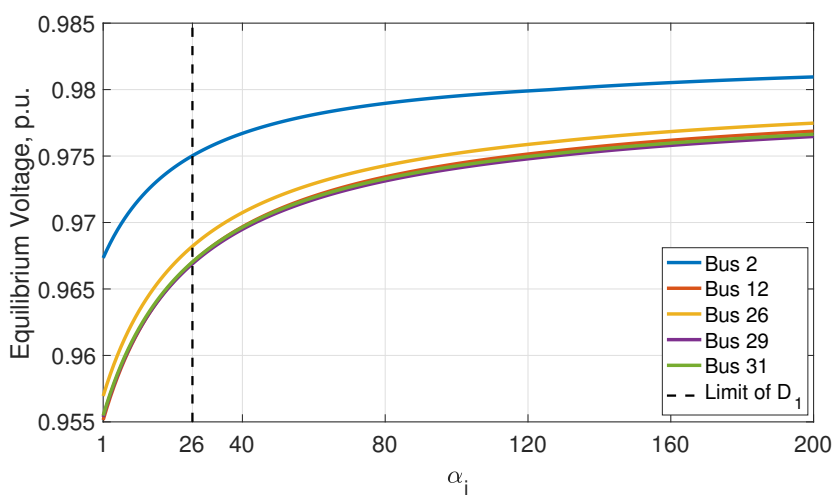

Fig. 5. Equilibrium voltage versus the $\alpha_{i}$ value: As $\alpha_{i}$ increases, the equilibrium voltage $v_{i}^{*}$ deviates less from the nominal value.

record the corresponding equilibrium voltages $v^{*}$. As shown in Fig. 5, $v^{*}$ gets closer to $v^{\text {nom }}$ as $\alpha_{i}$ increases. This confirms our previous discussion that steeper control functions are to be implemented to achieve smaller voltage deviations from the nominal value.

\section{B. Dynamics}

1) Convergence of Dynamical System $\mathcal{D}_{1}$ : As shown in Fig. 6, the dynamical system $\mathcal{D}_{1}$ displays less stable behavior as the control function become steeper with the increase of $\alpha_{i}$ value, till it ends up with oscillation when $\alpha_{i}$ becomes too large. See also the vertical dash line on Fig. 5 beyond which there is no convergence. ${ }^{2}$ As discussed in Section III-C, there is a contention between convergence and equilibrium performance for the non-incremental voltage control (12b): A smaller (absolute) slope is preferred for convergence, while a larger one is preferred for voltage regulation at the equilibrium.

2) Convergence of Dynamical Systems $\mathcal{D}_{2}$ and $\mathcal{D}_{3}$ : As discussed in Section IV, given any control function, $\mathcal{D}_{2}$ and $\mathcal{D}_{3}$ converge if small enough stepsizes are chosen, and we can

\footnotetext{
${ }^{2}$ The $\alpha_{i}$ value for the vertical dash line on Fig. 5 is obtained numerically. It is much larger than the sufficient but conservative condition $\alpha_{i}<9.96$ given by (24) or $\alpha_{i}<3.15$ given by (28).
}
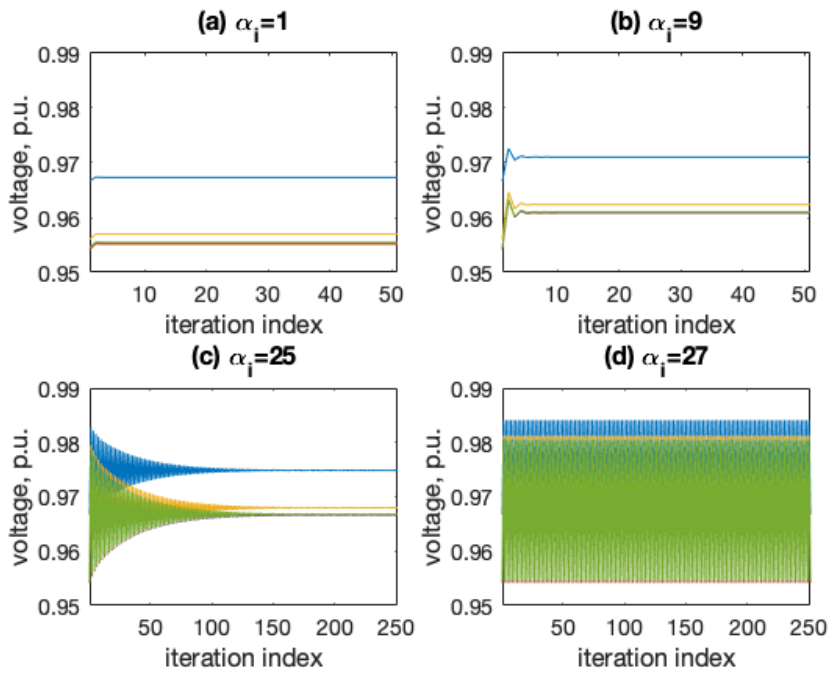

Fig. 6. Voltage convergence under dynamical system $\mathcal{D}_{1}$ with different slopes of the piecewise linear control function: voltage does not converge when the (absolute) slope of the control function become too large (when $\alpha_{i}>26$ in this example).

thus decouple the equilibrium property from the dynamical property. For instance, when $\alpha_{i}=27$, the dynamical system $\mathcal{D}_{1}$ does not converge; see Fig. 6(b). However, as shown in Fig. 7, when the stepsizes $\gamma_{2}$ and $\gamma_{3}$ are properly chosen, the dynamical systems $\mathcal{D}_{2}$ and $\mathcal{D}_{3}$ converge.

3) Convergence at Non-Differentiable Point: We have discussed in Section IV-A that the dynamical system $\mathcal{D}_{2}$ based on subgradient algorithm can only converge to within a small neighborhood of the equilibrium if it is at a nonsmooth point of the objective function (19). We tune the parameters such that the equilibrium reactive power provisioned at certain bus-bus 2 in this case-is close to zero. As shown in Fig. $8, \mathcal{D}_{2}$ eventually converges to a small region around the optimum, even with very small stepsize. On the other hand, as shown in Fig. 9, the dynamical system $\mathcal{D}_{3}$ based on pseudogradient algorithm converges to the equilibrium despite the non-smoothness of the objective function at the equilibrium. See the embedded windows in Fig. 8-9. 
(a) D2: $\gamma_{2}=30$

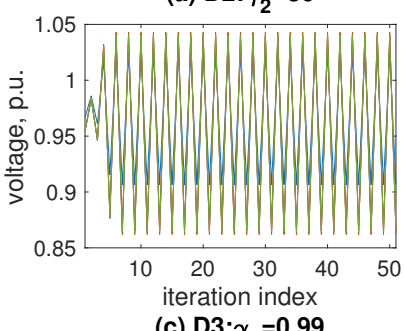

(c) D3: $\gamma_{3}=0.99$

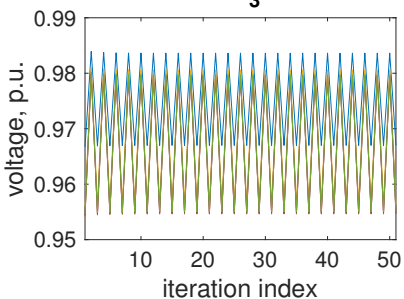

(b) D2: $\gamma_{2}=23$

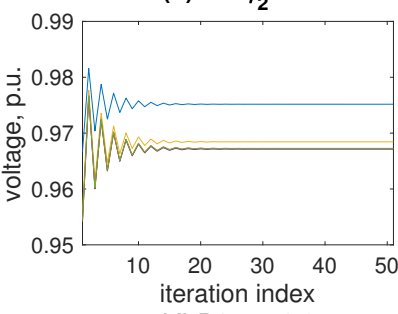

(d) D3: $\gamma_{3}=0.9$

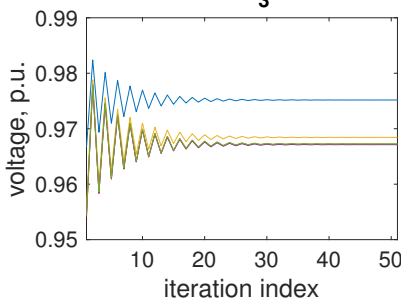

Fig. 7. Evolution of voltage of the dynamical systems $\mathcal{D}_{2}$ and $\mathcal{D}_{3}$ with $\alpha_{i}=27$ : Convergence is ensured with small enough stepsizes.

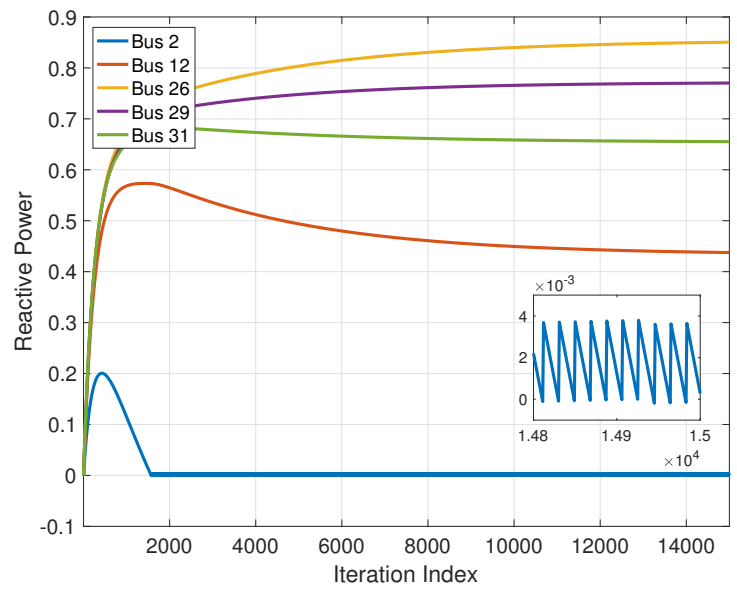

Fig. 8. Convergence of dynamical system $\mathcal{D}_{2}$ to within a small neighborhood of the equilibrium when $q_{2}^{*} \approx 0$.

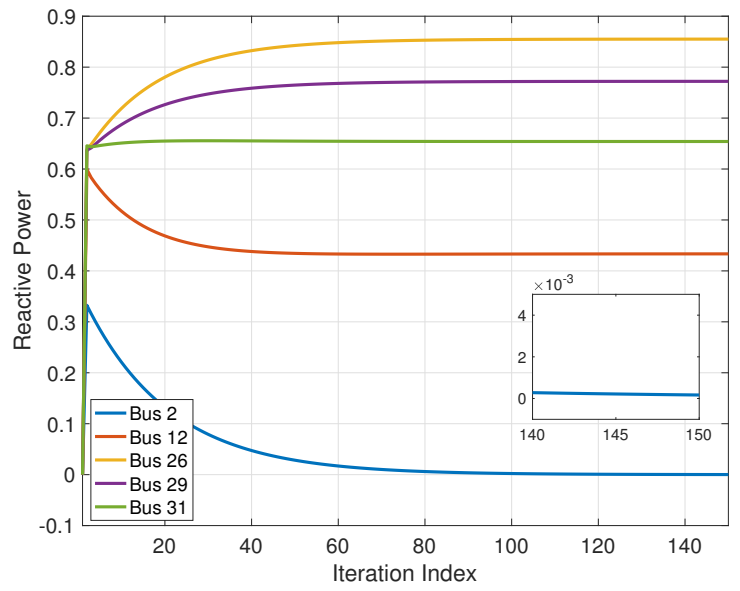

Fig. 9. Convergence of dynamical system $\mathcal{D}_{3}$ to the equilibrium point when $q_{2}^{*} \approx 0$.

\section{ConcLusion}

We have investigated local voltage control with a general class of control functions in distribution networks. We show that the power system dynamics with non-incremental local voltage control can be seen as a distributed algorithm for solving a well-defined optimization problem (reverse engineering). The reverse engineering further reveals a limitation of the non-incremental voltage control: the convergence condition is restrictive and prevents better voltage regulation at equilibrium. This motivates us to design two incremental local voltage control schemes based on different algorithms for solving the same optimization problem (forward engineering). The new control schemes decouple the dynamical property from the equilibrium property and have much less restrictive convergence conditions. This work presents another step towards developing a new foundation-network dynamics as optimization algorithms-for distributed real-time control and optimization of future power networks

\section{REFERENCES}

[1] M. E. Baran and F. F. Wu, "Optimal capacitor placement on radial distribution systems," IEEE Trans. on Power Delivery, vol. 4, no. 1, pp. 725-734, 1989.

[2] - "Optimal sizing of capacitors placed on a radial distribution system," IEEE Trans. on Power Delivery, vol. 4, no. 1, pp. 735-743, 1989.

[3] A. McCrone, U. Moslener, F. d'Estais, and C. Grüning, "Global trends in renewable energy investment," 2017.

[4] REN21, "Renewables 2017 global status report," 2017.

[5] Standards Coordinating Committee 21 of Institute of Electrical and Electronics Engineers, Inc., IEEE Standard, P1547.8 ${ }^{\mathrm{TM}} / \mathrm{D} 8$, "Recommended practice for establishing methods and procedures that provide supplemental support for implementation strategies for expanded use of IEEE Standard 1547," IEEE ballot document, Aug. 2014.

[6] V. Kekatos, G. Wang, A. J. Conejo, and G. B. Giannakis, "Stochastic reactive power management in microgrids with renewables," IEEE Trans. on Power Systems, vol. 30, no. 6, pp. 3386-3395, 2015.

[7] M. Farivar, C. R. Clarke, S. H. Low, and K. M. Chandy, "Inverter var control for distribution systems with renewables," Proc. of IEEE International Conference on Smart Grid Communications (SmartGridComm), pp. 457-462, 2011.

[8] J. Lavaei and S. H. Low, "Zero duality gap in optimal power flow problem," IEEE Trans. on Power Systems, vol. 27, no. 1, pp. 92-107, 2012.

[9] S. H. Low, "Convex relaxation of optimal power flow-Part I: Formulations and equivalence," IEEE Trans. on Control of Network Systems, vol. 1, no. 1, pp. 15-27, 2014.

[10] - "Convex relaxation of optimal power flow-Part II: Exactness," IEEE Trans. on Control of Network Systems, vol. 1, no. 2, pp. 177-189, 2014.

[11] V. Kekatos, L. Zhang, G. B. Giannakis, and R. Baldick, "Voltage regulation algorithms for multiphase power distribution grids," IEEE Trans. on Power Systems, vol. 31, no. 5, pp. 3913-3923, 2016.

[12] K. Baker, A. Bernstein, E. Dall'Anese, and C. Zhao, "Network-cognizant voltage droop control for distribution grids," IEEE Trans. on Power Systems, vol. 33, no. 2, pp. 2098-2108, 2017.

[13] E. Dall'Anese, S. V. Dhople, and G. B. Giannakis, "Optimal dispatch of photovoltaic inverters in residential distribution systems," IEEE Trans. on Sustainable Energy, vol. 5, no. 2, pp. 487-497, 2014.

[14] X. Zhou, E. Dall'Anese, L. Chen, and A. Simonetto, "An incentive-based online optimization framework for distribution grids," IEEE Trans. on Automatic Control, vol. 63, no. 7, pp. 2019-2031, 2017.

[15] X. Zhou, E. Dall'Anese, and L. Chen, "Online stochastic control of discrete loads in distribution grids," IEEE Trans. on Automatic Control, 2019.

[16] N. Li, G. Qu, and M. Dahleh, "Real-time decentralized voltage control in distribution networks," Proc. of Annual Allerton Conference on Communication, Control, and Computing (Allerton), pp. 582-588, 2014. 
[17] S. Bolognani and S. Zampieri, "A distributed control strategy for reactive power compensation in smart microgrids," IEEE Trans. on Automatic Control, vol. 58, no. 11, pp. 2818-2833, 2013.

[18] S. Magnússon, G. Qu, C. Fischione, and N. Li, "Voltage control using limited communication," IEEE Trans. on Control of Network Systems, vol. 6, no. 3, pp. 993-1003, 2019.

[19] Q. Peng and S. H. Low, "Distributed optimal power flow algorithm for radial networks, I: Balanced single phase case," IEEE Trans. on Smart Grid, vol. 9, no. 1, pp. 111-121, 2016.

[20] P. Šulc, S. Backhaus, and M. Chertkov, "Optimal distributed control of reactive power via the alternating direction method of multipliers," IEEE Trans. on Energy Conversion, vol. 29, no. 4, pp. 968-977, 2014.

[21] W. Shi, X. Xie, C. C. Chu, and R. Gadh, "Distributed optimal energy management in microgrids," IEEE Trans. on Smart Grid, vol. 6, no. 3 , pp. 1137-1146, 2015.

[22] S. Magnússon, P. C. Weeraddana, and C. Fischione, "A distributed approach for the optimal power-flow problem based on ADMM and sequential convex approximations," IEEE Trans. on Control of Network Systems, vol. 2, no. 3, pp. 238-253, 2015.

[23] M. Bazrafshan and N. Gatsis, "Decentralized stochastic optimal power flow in radial networks with distributed generation," IEEE Trans. on Smart Grid, vol. 8, no. 2, pp. 787-801, 2017.

[24] C. Wu, G. Hug, and S. Kar, "Distributed voltage regulation in distribution power grids: Utilizing the photovoltaics inverters," Proc. of American Control Conference (ACC), 2017, pp. 2725-2731, 2017.

[25] J. W. Simpson-Porco, F. Dörfler, and F. Bullo, "Voltage stabilization in microgrids via quadratic droop control," IEEE Trans. on Automatic Control, vol. 62, no. 3, pp. 1239-1253, 2017.

[26] H. Zhu and H. J. Liu, "Fast local voltage control under limited reactive power: Optimality and stability analysis," IEEE Trans. on Power Systems, vol. 31, no. 5, pp. 3794-3803, 2016.

[27] X. Zhou, J. Tian, L. Chen, and E. Dall'Anese, "Local voltage control in distribution networks: A game-theoretic perspective," Proc. of North American Power Symposium (NAPS), pp. 1-6, 2016.

[28] X. Zhou and L. Chen, "An incremental local algorithm for better voltage control in distribution networks," Proc. of IEEE Conference on Decision and Control (CDC), pp. 2396-2402, 2016.

[29] P. Jahangiri and D. C. Aliprantis, "Distributed Volt/VAr control by PV inverters," IEEE Trans. on Power Systems, vol. 28, no. 3, pp. 3429-3439, 2013.

[30] B. A. Robbins, C. N. Hadjicostis, and A. D. Domínguez-García, "A twostage distributed architecture for voltage control in power distribution systems," IEEE Trans. on Power Systems, vol. 28, no. 2, pp. 1470-1482, 2013.

[31] K. Turitsyn, P. Šulc, S. Backhaus, and M. Chertkov, "Options for control of reactive power by distributed photovoltaic generators," Proc. of the IEEE, vol. 99, no. 6, pp. 1063-1073, 2011.

[32] G. Cavraro, S. Bolognani, R. Carli, and S. Zampieri, "The value of communication in the voltage regulation problem," Proc. of IEEE Conference on Decision and Control (CDC), pp. 5781-5786, 2016.

[33] F. Andrén, B. Bletterie, S. Kadam, P. Kotsampopoulos, and C. Bucher, "On the stability of local voltage control in distribution networks with a high penetration of inverter-based generation," IEEE Trans. on Industrial Electronics, vol. 62, no. 4, pp. 2519-2529, 2015.

[34] G. Cavraro and R. Carli, "Local and distributed voltage control algorithms in distribution networks," IEEE Trans. on Power Systems, vol. 33, no. 2, pp. 1420-1430, 2017.

[35] R. Carli and G. Cavraro, "Algorithms for voltage control in distribution networks," Proc. of IEEE International Conference on Smart Grid Communications (SmartGridComm), pp. 737-742, 2015.

[36] B. Zhang, A. D. Dominguez-Garcia, and D. Tse, "A local control approach to voltage regulation in distribution networks," Proc. of IEEE North American Power Symposium (NAPS), pp. 1-6, 2013.

[37] L. Chen and S. You, "Reverse and forward engineering of frequency control in power networks," IEEE Trans. on Automatic Control, vol. 62, no. 9, pp. 4631-4638, 2017.

[38] N. Li, C. Zhao, and L. Chen, "Connecting automatic generation control and economic dispatch from an optimization view," IEEE Trans. on Control of Network Systems, vol. 3, no. 3, pp. 254-264, 2016.

[39] C. Zhao, U. Topcu, and S. Low, "Swing dynamics as primal-dual algorithm for optimal load control," Proc. of IEEE International Conference on Smart Grid Communications (SmartGridComm), pp. 570-575, 2012.

[40] X. Zhang and A. Papachristodoulou, "A real-time control framework for smart power networks with star topology," Proc. of American Control Conference (ACC), pp. 5062-5067, 2013.
[41] C. Zhao, U. Topcu, N. Li, and S. Low, "Design and stability of load-side primary frequency control in power systems," IEEE Trans. on Automatic Control, vol. 59, pp. 1177-1189, 2014

[42] E. Mallada and S. H. Low, "Distributed frequency-preserving optimal load control," IFAC Proceedings Volumes, vol. 47, no. 3, pp. 5411-5418, 2014.

[43] F. Dorfler, J. W. Simpson-Porco, and F. Bullo, "Breaking the hierarchy: Distributed control \& economic optimality in microgrids," IEEE Trans. on Control of Network Systems, vol. 3, no. 3, pp. 241-253, 2016.

[44] X. Zhou and L. Chen, "A new perspective to synchronization in networks of coupled oscillators: Reverse engineering and convex relaxation," IFAC-PapersOnLine, vol. 48, no. 22, pp. 40-45, 2015.

[45] M. E. Baran and F. F. Wu, "Network reconfiguration in distribution systems for loss reduction and load balancing," IEEE Trans. on Power Delivery, vol. 4, no. 2, pp. 1401-1407, 1989.

[46] E. Lakervi and E. J. Holmes, Electricity Distribution Network Design. Peter Peregrinus Ltd., 1995.

[47] Z. Liu, S. You, X. Zhou, G. Ding, and L. Chen, "Signal-anticipation in local voltage control in distribution systems," IEEE Trans. on Smart Grid, vol. 11, no. 1, pp. 233-246, 2020.

[48] S. Boyd and L. Vandenberghe, Convex Optimization. Cambridge University Press, 2004.

[49] J. Wen, Q. Wu, D. Turner, S. Cheng, and J. Fitch, "Optimal coordinated voltage control for power system voltage stability," IEEE Trans. on Power Systems, vol. 19, no. 2, pp. 1115-1122, 2004.

[50] R. S. Varga, Matrix iterative analysis. Springer Science \& Business Media, 2009, vol. 27.

[51] O. Galor, Discrete dynamical systems. Springer Science \& Business Media, 2007.

[52] R. D. Zimmerman, C. E. Murillo-Sánchez, and R. J. Thomas, "MATPOWER: Steady-state operations, planning, and analysis tools for power systems research and education," IEEE Trans. on Power Systems, vol. 26, no. 1, pp. 12-19, 2011.

[53] M. Farivar, L. Chen, and S. Low, "Equilibrium and dynamics of local voltage control in distribution systems," Proc. of IEEE Conference on Decision and Control (CDC), pp. 4329-4334, 2013.

[54] M. Farivar, X. Zhou, and L. Chen, "Local voltage control in distribution systems: An incremental control algorithm," Proc. of IEEE International Conference on Smart Grid Communications (SmartGridComm), pp. 732-737, 2015

[55] X. Zhou, M. Farivar, and L. Chen, "Pseudo-gradient based local voltage control in distribution networks," Proc. of IEEE Annual Allerton Conference on Communication, Control, and Computing (Allerton), pp. 173-180, 2015.

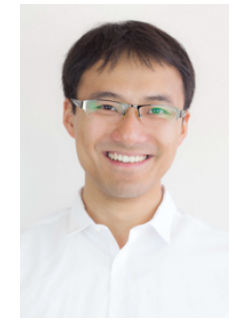

Xinyang Zhou (M'18) received the B.S. degree in Engineering from Zhejiang University, China, in 2012, and the Ph.D. degree in Telecommunications from the University of Colorado, Boulder, CO, USA, in 2018. He is currently a researcher with the $\mathrm{Na}$ tional Renewable Energy Laboratory, Golden, CO, USA. His current research interest is in distributed optimization and control for power systems.

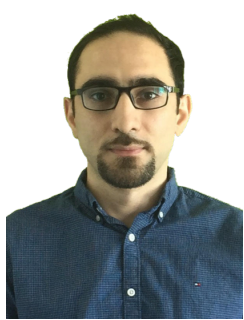

Masoud Farivar is currently a research scientist at Google, Mountain View. He received his Ph.D. in Electrical Engineering from California Institute of Technology in 2015, and a dual BSc. degree in Electrical Engineering and Computer Science from Sharif University of Technology in 2009. Masoud was a recipient of Amgen postdoctoral fellowship, and Resnick Institute fellowship for sustainability. His research interest broadly includes Machine Learning, Optimization, and Control. 


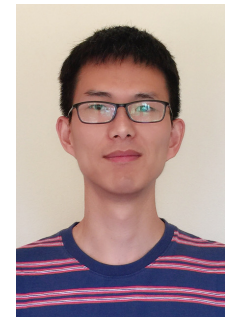

Zhiyuan Liu received a B.S. in Computer Science and Technology from University of Science and Technology of China (USTC), China in 2015. He is currently a $\mathrm{PhD}$ student in Computer Science at the University of Colorado at Boulder. His current research interest is in distributed optimization and control for networked systems.

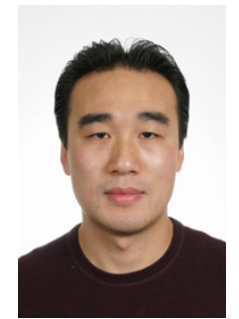

Lijun Chen (M'05) is an Assistant Professor of Computer Science at University of Colorado at Boulder. He received a Ph.D. in Control and Dynamical Systems from California Institute of Technology in 2007. He was a co-recipient of the Best Paper Award at the IEEE International Conference on Mobile Ad-hoc and Sensor Systems (MASS) in 2007. His current research interests include optimization and control of networked systems and autonomous systems, distributed optimization and control, machine learning and its integration with control, and game theory and its engineering application. He is an editor for the IEEE Transactions on Communications and editor for the IEEE Control Systems Society Conference Editorial Board. He serves as the chair of the Special Interest Group in ICT-Enabled Transactive Energy and Grid Economics of IEEE ComSoc Smart Grid Communications Emerging Technical Subcommittee 2017-2019.

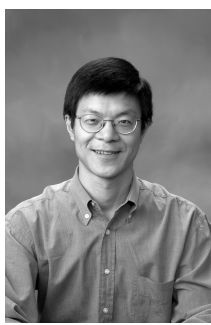

Steven H. Low (F'08) is a Professor of the Department of Computing \& Mathematical Sciences and the Department of Electrical Engineering at Caltech. Before that, he was with AT\&T Bell Laboratories, Murray Hill, NJ, and the University of Melbourne, Australia. He was a co-recipient of IEEE best paper awards and is an IEEE Fellow. His research on communication networks has been accelerating more than 1TB of Internet traffic every second since 2014. He was a member of the Networking and Information Technology Technical Advisory Group for the US President's Council of Advisors on Science and Technology (PCAST) in 2006. He received his B.S. from Cornell and $\mathrm{PhD}$ from Berkeley, both in EE. 